\title{
Liquid crystals in waveguides for tuning and sensing
}

\author{
Kristiaan Neyts, ${ }^{* 1}$ Wout Decort, ${ }^{1,2}$ Hamidreza Azarinia, ${ }^{1}$ Pieter Vanbrabant, ${ }^{1}$ Richard James, ${ }^{1}$ Jeroen Beeckman ${ }^{1}$ \\ ${ }^{1}$ ELIS Department, Ghent University, Sint-Pietersnieuwstraat 41, B-9000 Gent \\ ${ }^{2}$ INTEC Department, Ghent University, Sint-Pietersnieuwstraat 41, B-9000 Gent
}

Received February 14, 2011; accepted February 15, 2011; published March 31, 2011

\begin{abstract}
The orientation of liquid crystal in the neighborhood of an optical waveguide determines the propagation speed and the losses in the waveguide. This principle can be used for tuning the resonance frequency of a ring resonator or for sensing the presence of molecules at the interface between water and liquid crystal. In this work, experimental evidence and qualitative explanations are provided for these two cases.
\end{abstract}

In an optical waveguide, light is transported along some particular direction with low losses. The structure consists of a core material with a higher refractive index and an outside cladding with a lower refractive index, which provides one or more optical modes that do not couple to the outside world. In recent years many functionalities have been developed that allow the properties of the light that is transported in such optical waveguides to be altered. There exist band-pass filters, wavelength division multiplexers and splitters. Waveguides can also be used as sensors, because a change in the refractive index may be translated in a change in intensity or a shift in a resonance wavelength. Liquid crystals (LCs) can be used to influence the functionality of a waveguide, because liquid crystals are able to change their optical properties under the influence of a small voltage or a small surface effect. This can be used in tuning [1, 2] or sensing [3] applications.

There are many technologies used to realize waveguides and the contrast in the refractive index may be small (glass fibers, doped glass waveguides) or large (silicon on insulator waveguides - SOI). The larger the contrast, the better the light is guided by the waveguide. The liquid crystal can be present in the cladding of the waveguide or in the core.

Conventional electro-optical materials require high electric fields or strong currents to modify their optical properties. The reorientation of liquid crystals with their small elastic constant and strong anisotropy is ideal to modify the light propagation in an optical waveguide. In the past several groups have investigated the changing of the effective refractive index of waveguides by reorienting the liquid crystal in the neighbourhood of the waveguide core $[1,4-6]$.

\footnotetext{
*E-mail: kneyts@elis.ugent.be
}

Figure 1 illustrates the reorientation of a liquid crystal near a SOI waveguide before and after the application of a voltage. If the LC is initially oriented parallel with the waveguide [7], then the application of a potential difference between the silicon chip and an electrode above the chip will orient the LC molecules perpendicular to the surface. When the waveguide is a single mode TE waveguide, the fields are mainly along the $\mathrm{x}$ and $\mathrm{z}$ direction, as indicated in the figure. The contribution of the extra-ordinary index will be larger in the zero-volt case, because the LC is then parallel to the $\mathrm{z}$-component of the field. Switching the LC will therefore decrease the effective refractive index of the waveguide [8].
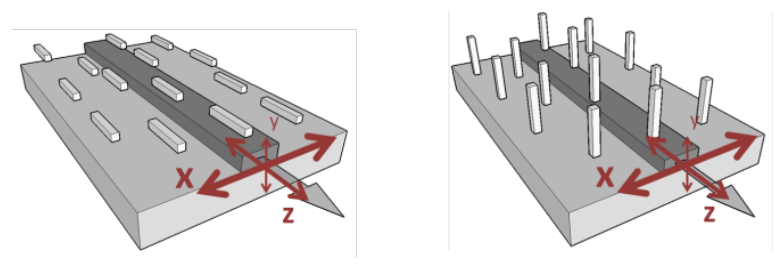

Fig. 1. Orientation of LC in the cladding of a TE silicon on an insulator waveguide, with indication of the electric field components of the mode. Left: orientation in the absence of a field. Right: orientation when a perpendicular field is applied.

Recently, we have realized another configuration with two strip electrodes patterned on a glass substrate, which are parallel to the waveguide [2]. The applied in-plane electric field is now perpendicular to the waveguide and can reorient the $\mathrm{LC}$ molecules along the $\mathrm{x}$-axis so that they are parallel to the strongest field component of the waveguide. In this case switching the LC increases the effective refractive index.
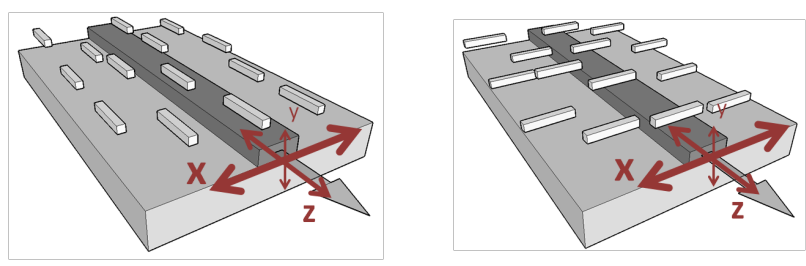

Fig. 2. Orientation of LC in the cladding of a TE silicon on an insulator waveguide, with indication of the electric field components the mode. Left: orientation in the absence of a field. Right: orientation when an in-plane field is applied. 
Both configurations have been realized, using the liquid crystal $5 \mathrm{CB}$. The waveguide is made in the form of a ring resonator with a radius of $4 \mu \mathrm{m}$, which is mode coupled to a straight waveguide. The electric field is either perpendicular to the substrate or in-plane, more or less homogeneous over the ring. Depending on the position in the ring, the field is parallel or perpendicular to the waveguide. In a ring resonator, the (average) change in the effective refractive index is proportional to the shift in the resonance wavelength. A decrease and an increase in the resonance wavelength have, indeed, been obtained for the cases of Fig. 1 and Fig. 2, respectively.

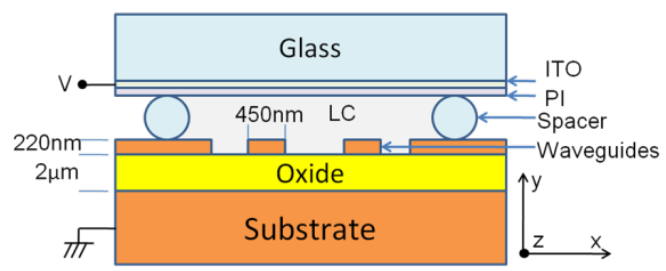

Fig. 3. Setup of the SOI waveguide with LC cladding [8]. This is the configuration corresponding to Fig. 1. For the configuration of Fig. 2 the ITO has the shape of a finger pattern.

In the above experiments the influence of the $\mathrm{LC}$ on the mode is rather limited because most of the light is confined in the high index silicon core and switching the LC modifies the effective refractive index by only a small fraction:

$$
\frac{\Delta n_{\text {eff }}}{n_{\text {eff }}}=\frac{\Delta \lambda}{\lambda} \square 10^{-3}
$$

In order to increase the influence of the LC on the light passing through a waveguide a completely different approach can be used: the LC is introduced in a small section in the core of a waveguide. For this approach, the use of SOI waveguides with their very high index contrast is undesirable because the beam expands very rapidly if the waveguide section is terminated. In order to reduce the expansion of the beam in the section with a liquid crystal, we have chosen a waveguide with a low refractive index contrast consisting of glass doped with a mixture of salt ions.

The fabrication of the device consists of the following steps: a blank glass substrate is covered by a thin aluminum mask with a slit of $10 \mu \mathrm{m}$; the substrate is immersed in a liquid bath of mixed nitrate salts for 45 minutes at $250^{\circ} \mathrm{C}$ to produce a slab waveguide; the mask is removed; a cavity of $20 \mu \mathrm{m}$ long, $140 \mu \mathrm{m}$ wide and $8 \mu \mathrm{m}$ deep is etched in the waveguide using a focused ion beam machine; finally the cavity is filled with liquid crystal 5CB.

In this approach, we are mainly interested in the fraction of the light that is transmitted through the LC in the cavity and captured by the waveguide at the other side. One possibility would be to change the transmission by reorienting the LC under the influence of an electric field. However, here we will investigate the reorientation under the influence of molecular interaction.

It is known that the presence of certain surfactants such as SDS in water can change the orientation of a liquid crystal near the LC/water boundary [9, 10]. In contact with pure water, the LC director will prefer to orient parallel to the common surface, as illustrated in Fig. 4 (left). When a sufficient concentration of SDS is present, the surfactant molecules will form a monolayer at the water/LC interface and the non-polar tails of the surfactant molecules favor the homeotropic alignment of the LC director (Fig. 4, right). The presence of a single monolayer of surfactant is apparently able to reorient LC molecules throughout the entire volume of the cavity. This makes the monolayer easily detectable by polarization microscopy, and if the area is sufficiently large, the monolayer can be visible to the naked eye. The polarization microscopy images in the middle of Fig. 4 show the reorientation of the LC caused by increasing the concentration of SDS to $0.4 \mathrm{mMolar}$ (on the right side). The cavity in Fig. 4 is $25 \mu \mathrm{m}$ by $40 \mu \mathrm{m}$ wide with a depth of $12.5 \mu \mathrm{m}$. The bottom and sides of the glass cavity have been treated with SE1211 in order to obtain the homeotropic alignment of the LC director.

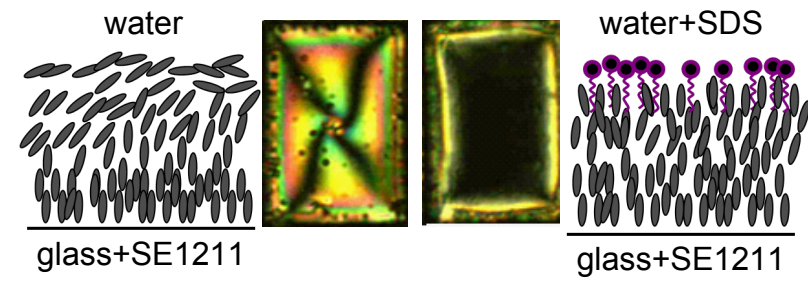

Fig. 4. Left: LC with a water overlay, inducing planar alignment. Right: LC with water overlay containing SDS, inducing homeotropic alignment. Middle: polarization microscopy images for LC filled cavity covered with low and high concentration of SDS.

The simulation of the director orientation in a rectangular cuboid with given boundary conditions has been carried out based on available software [11, 12]. Five walls have strong homeotropic anchoring and the top face has either planar or homeotropic anchoring. The simulation is carried out for a volume of $25 \mu \mathrm{m}$ by $40 \mu \mathrm{m}$ with a depth of $16.5 \mu \mathrm{m}$ and the LC parameters of 5CB. The result of this simulation is given in Fig. 5 [9]. For homeotropic alignment at the top, the director is vertical in most of the volume, and the transmission for crossed polarizers is low. For planar alignment at the top, the situation is more complex and there is a -1 hyperbolic point defect at the top surface. The two images in the middle show the simulated transmission patterns, when the LC in the cavity is observed with crossed polarizers oriented at $\pm 45^{\circ}$. 


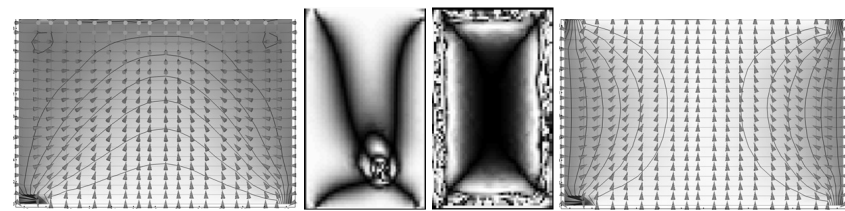

Fig. 5. Simulated director orientation in a vertical section (outside) and transmission profile (middle, with crossed polarizers at $45^{\circ}$ ) for LC in a cavity. Five walls have homeotropic alignment [9]. The top surface boundary condition is either planar (left side) or homeotropic (right side).

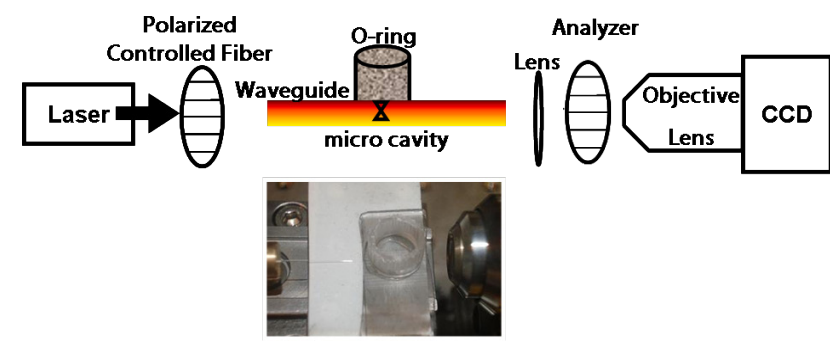

Fig. 6. Setup for measuring light passing through an LC filled cavity that intersects a glass optical waveguide. Top: schematic drawing. Bottom: photograph with lensed fiber, glass substrate and objective lens.

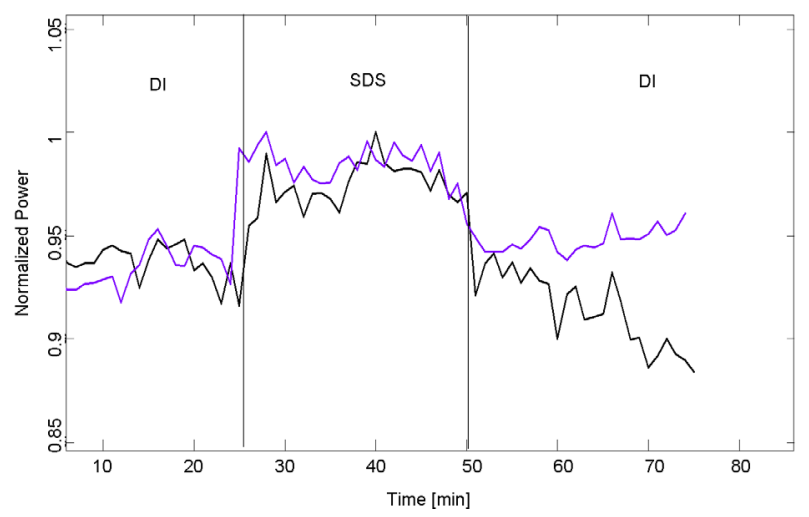

Fig. 7. Laser power detected at the exit face of the waveguide as a function of time. Initially, there is only DI water over the LC; after $25 \mathrm{~min}$ SDS is added to the solution and after $50 \mathrm{~min}$ the SDS is flushed with DI water.

Finally, we use the previously introduced glass-based waveguide with low refractive index contrast in combination with the cavity to enable the integrated detector of SDS in a waveguide based system. The scheme of the setup is illustrated in Fig 6. TE polarized laser light from a lensed fiber $(3 \mathrm{~mW})$ is coupled to the glass waveguide through a polished glass face. The waveguide enters the LC-filled microcavity from the side and light is collected by a second waveguide at the other side of the cavity. The emission from the exit face is imaged with a microscope objective and measured by integrating the intensity of the CCD image.
The setup is used to detect the presence of SDS over the LC in an integrated setup. Initially, the LC in the microcavity is in contact with pure water. Then a solution of DI water with $1 \mathrm{mM}$ SDS is introduced over the LC and an increase in the transmitted power is observed with the CCD camera. After 50min the SDS is flushed by adding an excess of DI water over the LC filled microcavity. The measured curve in Fig. 7 reveals that the transmitted power increases by adding SDS (orienting the LC perpendicular to the interface with water) and reduces to the initial level after flushing with DI water. This means that the SDS is desorbed by the excess of water.

In conclusion, we have demonstrated that the presence of a liquid crystal in the neighbourhood of a waveguide (in the cladding or in the core) can be used to modify the propagation speed and/or transmission of the light. This can be used in telecom or biodetection applications.

The authors acknowledge the support of IWT Vlaanderen, FWO-Vlaanderen, the Belgian IAP project project Photonics@be. The authors are part of the Center for Nano and BioPhotonics at UGent.

\section{References}

[1] B. Maune, R. Lawson, C. Gunn, A. Scherer, L. Dalton, Appl. Phys. Lett. 83, 4689 (2003).

[2] W. De Cort et al., J. Opt. Soc. Am. B 28, 79 (2011)

[3] C. Veilleux, R.J. Black, J. Lapierre, L.W. Reeves, J. Appl. Phys. 67, 6648 (1990).

[4] A. Di Falco,G. Assanto, Opt. Comm. 279, 210 (2007).

[5] J. Beeckman et al., J. Lightwave Techn. 27, 3812 (2009).

[6] Y. Xu, M.A. Uddin, P.S. Chung, H.P. Chan, Opt. Exp. 17, 7837 (2009).

[7] H. Desmet, K. Neyts, R. Baets, Integr. Opt. Silicon Photon. Photon. Integr. Circ. 6183, Z1831, 480 (2006).

[8] W. De Cort et al., Opt. Lett. 34, 2054 (2009).

[9] H. Azarinia et al., J. Appl. Phys. 106, 063101 (2009).

[10] V.K. Gupta, J.J. Skaife, T.B. Dubrovsky, N.L. Abbott, Science 279, 2077 (1998).

[11] R. James, E. Willman, F.A. Fernandez, S.E. Day, IEEE Trans. Magnet. 44, 814 (2008).

[12] F.A. Fernandez, S.E. Day, P. Trwoga, H.F. Deng, R. James, Mol. Cryst. Liq. Cryst. 375, 291 (2002). 